\title{
WHEN IS THE ALGEBRA OF REGULAR SETS FOR A FINITELY ADDITIVE BOREL MEASURE A $\sigma$-ALGEBRA?
}

\author{
THOMAS E. ARMSTRONG
}

(Received 4 May 1981; revised 3 December 1981)

Communicated by J. B. Miller

\begin{abstract}
It is shown that the algebra of regular sets for a finitely additive Borel measure $\mu$ on a compact Hausdorff space is a $\sigma$-algebra only if it includes the Baire algebra and $\mu$ is countably additive on the $\sigma$-algebra of regular sets. Any infinite compact Hausdorff space admits a finitely additive Borel measure whose algebra of regular sets is not a $\sigma$-algebra. Although a finitely additive measure with a $\sigma$-algebra of regular sets is countably additive on the Baire $\sigma$-algebra there are examples of finitely additive extensions of countably additive Baire measures whose regular algebra is not a $\sigma$-algebra. We examine the particular case of extensions of Dirac measures. In this context it is shown that all extensions of a $\{0,1\}$-valued countably additive measure from a $\sigma$-algebra to a larger $\sigma$-algebra are countably additive if and only if the convex set of these extensions is a finite dimensional simplex.

1980 Mathematics subject classification (Amer. Math. Soc.): 28 C 15, 28 A 60, 54 G 10.

Keywords: Borel measure, regularity, extensions of measures, completion regular compact space, Borel regular compact space.
\end{abstract}

\section{Introduction and synopsis}

In [16], Kupka noted that if a vector-valued Borel measure on a compact Hausdorff space $X$ is countably additive then its algebra of regular sets is in fact a $\sigma$-algebra. In Question 3.3.1 of [16], he asked whether countable additivity is necessary for this result. We essentially answer this question in the negative but do show that a good deal of countable additivity is implicit in the assumption that the algebra of regular sets of a finitely additive Borel measure is a $\sigma$-algebra. More specifically we show that, on any $\sigma$-algebra contained in the algebra of regular

(c) Copyright Australian Mathematical Society 1982 
sets of a finitely additive Borel measure $\mu, \mu$ is countably additive (Lemma 1 ). If in fact the algebra of regular sets for $\mu$ is a $\sigma$-algebra then it includes the $\mu$-completion of the Baire algebra and $\mu$ agrees with the canonical regular extension of $\mu$ to the Borel algebra from the Baire algebra at least on the $\sigma$-algebra of regular sets (Propositions 3 and 5). In fact, the latter statement holds even if $\mu$ is only assumed to be countably additive on the Baire algebra but with the algebra of regular sets not necessarily a $\sigma$-algebra. Corollary 3.1 answers Kupka's question affirmatively for completion regular compact Hausdorff spaces. Here a finitely additive Borel measure is countably additive if and only if its algebra of regular sets is $\sigma$-algebra. Corollary 3.2 shows that on any infinite compact Hausdorff space there is a finitely additive Borel measure which does not have a $\sigma$-algebra of regular sets. This follows from Proposition 4 which asserts that a Boolean algebra admits a non-countably additive measure if and only if it is not Cantor separable if and only if its Stone space is not an almost $P$-space, a result of independent interest.

The latter part of the paper examines the regular algebras of finitely additive Borel measures $\mu$ whose restriction to the Baire algebra is countably additive when $\mu$ is $\{0,1\}$-valued on the Baire algebra. Proposition 6 deals with the convex compact set of all extensions of a countably additive $\{0,1\}$-valued measure $\delta$ on a $\sigma$-algebra $\Sigma_{1}$ to a larger $\sigma$-algebra $\Sigma_{2}$. It is shown that this convex compact set is finite dimensional if and only if all extensions of $\delta$ are countably additive. Otherwise, there exist $2^{c}$ mutually singular non-atomic purely finitely additive extensions or $c\{0,1\}$-valued extensions where $c=2^{\aleph_{0}}$, (Corollary 6.1). This is applied to the case where $\Sigma_{1}$ is the Baire algebra, $\Sigma_{2}$ is the Borel algebra and $\delta$ is $\delta_{x}$ for some non- $G_{\delta}$-point $x \in X$. If the extensions of $\boldsymbol{\delta}_{x}$ to the Borel algebra are all countably additive there is a countably additive extension $\mu$ whose regular algebra is just the $\delta_{x}$-completion of the Baire algebra. However, for this to be true $X$ must be topologically pathological near $x$.

We conclude with an example which yields finitely additive Borel measures whose regular algebras are not $\sigma$-algebras yet contain the Baire algebra. If real valued measurable cardinals exist an example is given of a countably additive Borel measure whose regular $\sigma$-algebra is properly contained in the Borel algebra and properly contains the completed Baire algebra.

\section{When is the algebra of regular sets for a finitely additive Borel measure a $\sigma$-algebra?}

$\mathscr{B}_{0}$ and $\mathscr{B}$ denote, respectively, the Baire and Borel $\sigma$-algebras on $X$. $\mathcal{C}(X)$ denotes the real continuous functions on $X$ and $\mathscr{N}(X)$ the dual of $\mathcal{C}(X)$. $\Re(X)$ is identified, as usual, with both $C A\left(\mathscr{B}_{0}\right)$ the countable additive Baire measures 
and with $C A_{t}(\Re)$ the regular countably additive Borel measures. For any Boolean algebra $Q, B A(\mathbb{Q})$ denotes the finitely additive real measures of bounded variation on $\mathbb{Q}$ with $C A(\mathbb{Q})$ the band of countably additive elements of $B A(\mathbb{Q})$. If $\mu \in$ $B A^{+}(\mathscr{B})$ we denote by $\operatorname{Reg}(\mu)$ all $A \in \mathscr{B}$ so that $\inf \{\mu(\theta \backslash K): K$ compact $\subset A$ $\subset \theta$ open $\}=0$. Note that $\operatorname{Reg}(\mu)$ is an algebra which is $\mu$-complete in $\mathscr{B}$ in that whenever $\left\{A_{n}\right\}$ is an increasing sequence and $\left\{B_{n}\right\}$ is a decreasing sequence in $\operatorname{Reg}(\mu)$ with $A_{n} \subset B_{n}$ for all $n$ and with $\lim _{n \rightarrow \infty} \mu\left(B_{n} \backslash A_{n}\right)=0$ then $A \in \operatorname{Reg}(\mu)$ provided $A \in \mathscr{B}$ and $A_{n} \subset A \subset B_{n}$ for all $n$. For any algebra $\mathscr{Q} \subset \mathscr{B}$, $\hat{\mathbb{Q}}^{\mu}$ will denote its completion in $\mathscr{G}$ with respect to the finitely additive Borel measure $\mu$. Thus, $\operatorname{Reg}(\mu)=\left(\operatorname{Reg}(\mu)^{\mu}\right.$. This lemma was pointed out by Douglas Dokken. It is a generalization of Problem 7 on page 11 of [6].

Lemma 1. If $\Sigma$ is a o-algebra contained in $\operatorname{Reg}(\mu)$ for $\mu \in B A^{+}(\mathscr{B})$ then $\mu$ is countably additive on $\Sigma$.

Proof. It must be shown that if $\left\{D_{n}\right\} \subset \Sigma$ is a disjoint sequence with union $D$ then $\mu(D)=\sum_{n=1}^{\infty} \mu\left(D_{n}\right)$. That $\mu(D) \geqslant \sum_{n=1}^{\infty} \mu\left(D_{n}\right)$ is immediate. If we show that $\mu(D) \leqslant \sum_{n=1}^{\infty} \mu\left(D_{n}\right)+\varepsilon$ for any $\varepsilon>0$ the assertion will be established. Pick $K$ compact $\subset D$ with $\mu(D) \leqslant \mu(K)+\varepsilon / 2$. Pick $\theta_{n}$ open with $D_{n} \subset \theta_{n}$ and with $\mu\left(\theta_{n} \backslash D_{n}\right) \leqslant \varepsilon 2^{-n-1}$. Since $K \subset D \subset \cup_{n=1}^{\infty} \theta_{n}$ there is an integer $m$ so that $K \subset \theta_{1}$ $\cup \cdots \cup \theta_{m}$. For this $m$ it is true that $\mu(K) \leqslant \sum_{n=1}^{\infty} \mu\left(\theta_{m}\right) \leqslant \sum_{n=1}^{\infty} \mu\left(\theta_{n}\right)<$ $\sum_{n=1}^{\infty} \mu\left(D_{n}\right)+\varepsilon / 2$. Thus, $\mu(D)<\sum_{n=1}^{\infty} \mu\left(D_{n}\right)+\varepsilon$.

Remark. Lemma 1 is a consequence of Proposition 1.6 in Chapter V of [4] and of Lemma 1 of [25].

Corollary 1.1. a) If $\mu \in B A^{+}(\mathscr{B})$ and $\operatorname{Reg}(\mu)$ is a o-algebra then $\mu$ is countably additive on $\operatorname{Reg}(\mu)$.

b) $\operatorname{Reg}(\mu)$ is a $\sigma$-algebra if and only if $\mu$ is countably additive on the $\sigma$-algebra generated by $\operatorname{Reg}(\mu)$.

Proof. Only b) needs to be established. This is done in the standard fashion. Let $\left\{D_{n}\right\}$ be a disjoint sequence in $\operatorname{Reg}(\mu)$ with union $D$. Let $\theta_{n}$ be open with $D_{n} \subset \theta_{n}$ and $\mu\left(\theta_{n} \backslash D_{n}\right) \leqslant 2^{-n-1}$. $\varepsilon$ for a given $\varepsilon>0$. Let $m$ be such that $\mu\left(\cup_{n=m+1}^{\infty} D_{n}\right) \leqslant \varepsilon / 4$. Let $K_{n} \subset D_{n}$ for $n=1, \ldots, m$ be compacts with $\mu\left(D_{n} \backslash K_{n}\right)$ $<\frac{1}{4} \varepsilon m^{-1}$. We have $\mu\left[\left(\cup_{n=1}^{\infty} \theta_{n}\right) \backslash\left(\cup_{n=1}^{m} K_{n}\right)\right] \leqslant \varepsilon$ with $\cup_{n=1}^{m} K_{n} \subset D \subset \cup_{n=1}^{\infty} \theta_{n}$. Thus, $D \in \operatorname{Reg}(\mu)$. Thus, $\operatorname{Reg}(\mu)$ is a $\sigma$-algebra if $\mu$ is countably additive on the $\sigma$-algebra generated by $\operatorname{Reg}(\mu)$. The converse follows from a). 
Lemma 2. Let $A \in \operatorname{Reg}(\mu)$.

i) There exists $a G_{\delta} A_{\delta} \in \operatorname{Reg}(\mu)$ and an $F_{\sigma} A_{\sigma} \in \operatorname{Reg}(\mu)$ with $A_{\sigma} \subset A \subset A_{\delta}$ and $\mu\left(A_{\delta} \backslash A_{\sigma}\right)=0$.

ii) There exists a $G_{\delta} A^{\delta} \in \mathscr{B}_{0} \cap \operatorname{Reg}(\mu)$ and an $F_{\sigma} A^{\sigma} \in \mathscr{B}_{0} \cap \operatorname{Reg}(\mu)$ with $A_{\sigma} \subset A^{\sigma} \subset A^{\delta} \subset A_{\delta}$.

iii) $\mu(A)=\mu\left(A_{\sigma}\right)=\mu\left(A_{\delta}\right)=\mu\left(A^{\sigma}\right)=\mu\left(A^{\delta}\right)=\sup \{\mu(K): K$ compact Baire $\left.\subset A^{\sigma}\right\}=\inf \left\{\mu(G): G\right.$ open Baire $\left.\supset A^{\delta}\right\}$.

iv) There is an $A_{0} \in \mathscr{B}_{0} \cap \operatorname{Reg}(\mu)$ with $\mu\left(A \Delta A_{0}\right)=0$.

\section{ProOF.}

i) Immediate from the definition of regularity.

ii) Let $A_{\sigma}=\cup_{n=1}^{\infty} K_{n}$ and $A_{\sigma}=\cap G_{n}$ where $K_{n}$ is compact and $G_{n}$ is open for all $n$. By Urysohn's Theorem there is a compact $G_{\delta}, K_{n, m}^{\prime}$ satisfying $K_{n} \subset K_{n, m}^{\prime} \subset$ $G_{m}$ for all $n, m$. Set $K_{n}^{\prime}=\bigcap_{m=1}^{\infty} K_{n, m}^{\prime} . K_{n}^{\prime}$ is a compact $G_{\delta}$ and $K_{n} \subset K_{n}^{\prime} \subset A_{\delta}$ for all $n$. Set $A^{\sigma}$ equal to the $F_{\sigma}, \cup_{n=1}^{\infty} K_{n}^{\prime} . A^{\delta}$ is obtained analogously as a countable intersection of open $F_{\sigma}$ sets.

iii) From the definition of regularity the $K_{n}$ in ii) may be chosen with $\mu(A)=\sup \mu\left(K_{n}\right) \leqslant \sup \mu\left(K_{n}^{\prime}\right) \leqslant \sup \left\{\mu(K): K\right.$ compact Baire $\left.\subset A^{\sigma}\right\} \leqslant \mu\left(A^{\sigma}\right)$ $=\mu(A)$. Thus, $\mu(A)=\sup \left\{\mu(K): K\right.$ compact Baire $\left.\subset A^{\circ}\right\}$. Similarly, $\mu(A)=$ $\inf \left\{\mu(G): G\right.$ open Baire $\left.\supset A^{\sigma}\right\}$.

iv) Set $A_{0}=A^{\delta}$ or $A^{\sigma}$.

Plachky, [20], shows that if $\nu$ is a finitely additive probability on a Boolean algebra $\mathscr{Q}_{1}$ and $B A_{1}^{+}\left(Q_{1}, \nu, \mathbb{Q}_{2}\right)$ denotes the convex compact set of extensions of $\nu$ to a probability measure on a larger algebra $\mathscr{Q}_{2}$ then $\mu \in B A_{1}^{+}\left(\mathbb{Q}_{1}, \nu, Q_{2}\right)$ is extreme if and only if for all $A_{2} \in Q_{2}$ and $\varepsilon>0$ there is an $A_{1} \in Q_{1}$ with $\mu\left(A_{1} \Delta A_{2}\right)<\varepsilon$. Thus, in Lemma $2, \mu$, on $\operatorname{Reg}(\mu)$, is an extreme extension of its restriction to $\Re_{0} \cap \operatorname{Reg}(\mu)$.

Proposition 3. If $\mu \in B A^{+}(\mathscr{B})$ is such that $\operatorname{Reg}(\mu)$ is a $\sigma$-algebra then $\mathscr{B}_{0} \subset \operatorname{Reg}(\mu)$.

To establish this we first consider the case $X=[0,1]$. Let $Y$ denote those $x \in(0,1)$ so that $\inf \{\mu(\theta): x \in \theta$ open $\}=0$. The complement of $Y$ is at most countably hence $Y$ is dense. Each $\{x\}$ with $x \in Y$ is in $\operatorname{Reg}(\mu)$ with $\mu(\{x\})=0$. For $\varepsilon>0$ let $\theta$ be an open set containing $x \in Y$ with $\mu\left(\theta_{\varepsilon}\right)<\varepsilon, K_{\varepsilon}^{-}=[0, x) \backslash \theta_{\varepsilon}$ and $K_{\varepsilon}^{+}=(x, 1] \backslash \theta_{\varepsilon}$. Both $K_{\varepsilon}^{-}$and $K_{\varepsilon}^{+}$are compact. It is easily verified that $\lim _{\varepsilon \rightarrow 0} \mu\left(K_{\varepsilon}^{-}\right)=\mu([0, x))$ and $\lim _{\varepsilon \rightarrow 0} \mu\left(K_{\varepsilon}^{+}\right)=\mu((x, 1])$. Thus, $\{[0, x),(x, 1]\} \subset$ $\operatorname{Reg}(\mu)$. It follows that all intervals, open, closed, or half open, whose endpoints 
are chosen from $Y$ belong to $\operatorname{Reg}(\mu)$. The $\sigma$-algebra generated by these intervals is

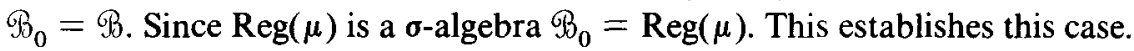

Let $X$ be arbitrary and let $f: X \rightarrow[0,1]$ be continuous. Let $\nu$ be the finitely additive Borel measure on $[0,1]$ which is the image of $\mu$ under $f$. Thus, for Borel $A \subset[0,1], \nu(A)=\mu\left(f^{-1}(A)\right)$. Just as in the countably additive case $A \in \operatorname{Reg}(\nu)$ if and only if $f^{-1}(A) \in \operatorname{Reg}(\mu)$. Consequently, $\operatorname{Reg}(\nu)$ is a $\sigma$-algebra hence is equal to the Borel algebra of $[0,1]$ by the special case just established. Thus, $f$ is measurable for the $\sigma$-algebra $\operatorname{Reg}(\mu)$. Since $f$ is arbitrary it follows that all $f \in \mathcal{C}(X)$ are $\operatorname{Reg}(\mu)$-measurable. Thus, since $\mathscr{B}_{0}$ is the smallest $\sigma$-algebra so that all $f \in \mathcal{C}(X)$ are $\mathscr{G}_{0}$-measurable, $\mathscr{B}_{0} \subset \operatorname{Reg}(\mu)$. This establishes the proposition.

In [4], Babiker and Knowles define a space $X$ to be completion regular if and only if every $\mu \in C A^{+}\left(\mathscr{B}_{0}\right)$ is completion regular in the sense of Berberian [5]. That is, each $\mu \in C A^{+}\left(\mathscr{B}_{0}\right)$ has a unique extension in $B A^{+}(\mathscr{B})$. Alternatively $X$ is completion regular if and only if $\mathscr{B}$ is the $\mu$-completion of $\mathscr{B}_{0}$ for all $\mu \in C A^{+}\left(\mathscr{B}_{0}\right)$. Examples of completion regular spaces include all perfectly normal compact Hausdorff spaces $X$. In [5] Berberian notes that if $X$ is completion regular all points must be $G_{\delta}$ 's. Under the assumption that the continuum is real valued measurable an example may be constructed of a non-completion regular $X$ each of whose points is a $G_{\delta}$. In order that $X$ be completion regular it is necessary and sufficient that every Borel set be regular with respect to the paving of compact $G_{\delta}$ 's for all countably additive Borel measures. This corollary is easily deduced from the definition of completion regularity.

COROLlaRY 3.1. Let $X$ be completion regular. The following are equivalent for $\mu \in B A^{+}(\mathscr{B})$
a) $\operatorname{Reg}(\mu)$ is a $\sigma$-algebra
b) $\operatorname{Reg}(\mu)=\mathscr{B}$
c) $\mu \in C A^{+}(\mathscr{B})=C A_{t}^{+}(\mathscr{B})$.

Corollary 3.2. If $X$ is an infinite compact Hausdorff space there is a $\mu \in B A^{+}$ ( 9$)$ so that $\operatorname{Reg}(\mu)$ is not a $\sigma$-algebra.

Proof. Any extension $\mu$ to $\mathscr{B}$ of a member of $B A^{+}\left(\mathscr{B}_{0}\right) \backslash C A^{+}\left(\mathscr{B}_{0}\right)$ will do. The non-emptiness of $B A^{+}\left(\mathscr{B}_{0}\right) \backslash C A^{+}\left(\mathscr{B}_{0}\right)$ is a special case of Proposition 4.

We are interested in determining for which infinite Boolean algebras $\mathbb{Q}$ every element of $\mathrm{BA}^{+}(Q)$ is countably additive. If no infinite strictly decreasing sequence in $\mathscr{Q}$ has a lower bound then, automatically, $B A^{+}(\mathscr{Q})=C A^{+}(\mathscr{Q})$. Such Boolean algebras are termed Cantor separable in [28]. Cantor separable Boolean algebras $\Theta$ are characterized in terms of their Stone space $X_{\mathbb{P}}$ by the fact that each 
non-empty zero set has a non-empty interior. Completely regular spaces $X$ with the aforementioned property are called almost $P$-spaces in [17] and have been studied in [7], [10] and [27]. Thus, $Q$ is Cantor separable if $X_{\mathfrak{Q}}$ is an almost $P$-space. Notice that if $\mathscr{Q}$ is $\sigma$-complete it is not Cantor separable if it is infinite. $\beta N \backslash N$ is the most familiar example of an almost $P$-space $[9,65.8]$. Graves and Wheeler in [10] give a method for producing a large class of almost $P$-spaces. The following proposition was pointed out by R. F. Wheeler.

Proposition 4. The following are equivalent for an infinite Boolean Algebra $氏$

a) $\mathcal{Q}$ is Cantor separable

b) $X_{\mathbb{Q}}$ is an almost $P$-space

c) $B A^{+}(Q)=C A^{+}(\mathbb{Q})$.

Proof. We already have a) $\Leftrightarrow b) \Rightarrow c$ ). Let us assume c) and see that this implies b). Notice that all $\{0,1\}$-valued elements of $B A^{+}(\mathbb{Q})$ are countably additive. Phrased in terms of the corresponding ultrafilters on $\mathbb{Q}$ this says that if $\left\{A_{n}: n \in \mathbf{N}\right\}$ is a decreasing sequence in an ultrafilter then $\varnothing \neq \inf _{n} A_{n}$. That is, there is an $A_{\infty} \in \mathbb{Q}$ with $\varnothing \neq A_{\infty} \subset A_{n}$ for all $n$. Since every decreasing sequence of non-empty elements of $\mathscr{U}$ lies in an ultrafilter this says that no decreasing sequence of non-empty elements of $\mathscr{Q}$ has $\varnothing$ as infimum. In particular, regarding $\mathbb{Q}$ as the clopen algebra of $X_{\mathfrak{E}}$, the intersection of a decreasing sequence of non-empty clopen sets (that is, a zero set) has non-empty interior. Thus, c) implies both a) and b).

REMARK. We use the term $\delta$-ultrafilter for an arbitrary Boolean algebra to denote any ultrafilter whose corresponding $\{0,1\}$-valued measure is countably additive.

A compact Hausdorff space $X$ is called Borel regular [19], or Radon, [21], if and only if $C A^{+}(\mathscr{B})=C A_{t}^{+}(\mathscr{B})$ if and only if every $\mu \in C A^{+}\left(\mathscr{B}_{0}\right)$ has a unique extension, the regular extension, to $\mathscr{B}$ belonging to $C A^{+}(\mathscr{B})$. If $\mu \in C A_{1}^{+}(\mathscr{B}) \backslash$ $C A_{t}^{+}(B)$ then $\operatorname{Reg}(\mu)$ is a super- $\sigma$-algebra of $\mathscr{B}_{0}$ properly contained in $\mathscr{B}$. The canonical example of a non-Borel regular space is the compact ordinal space $\left[0, \omega_{1}\right]$ where $\omega_{1}$ is the first uncountable ordinal. There are countably additive $\{0,1\}$-valued extensions of the Dirac measure $\delta_{\omega_{1}}$ from $\mathscr{B}_{0}$ to $\mathscr{B}$ other than the regular extension [9, ex. 53.10a]. An example of a Borel regular space $X$ which is not completion regular is the one point compactification $D \cup\{\infty\}$ of a discrete space $D$ with uncountable non-real-valued measurable cardinal, [8, ex. 6.2]. The Dirac measure $\delta_{\infty}$ has extensions from $\mathscr{B}_{0}$ to $\mathscr{B}$ other than the regular one but all must be purely finitely additive [2], [13], since they induce on $D$ finitely additive, 
diffuse [2], probability measures. We shall be primarily concerned with $\operatorname{Reg}(\mu)$ for $\mu$ non-countably additive yet with $\mu$ countably additive on $\mathscr{B}_{0}$ but occasionally with $\mu$ countably additive and non-regular on $\mathscr{B}$. In any case, $\mu_{\text {reg }}$ will denote the unique element of $C A_{t}^{+}(\mathscr{B})$ agreeing with $\mu$ on $\Re_{0}$.

Proposition 5. Let $\mu \in B A^{+}(\mathscr{B})$ be countably additive on $\mathscr{B}_{0}$. On $\operatorname{Reg}(\mu), \mu$ and $\mu_{\text {reg }}$ coincide.

Proof. Let $A \in \operatorname{Reg}(\mu)$. One can, in the proof of Lemma 2, find $A_{\sigma}$ an $F_{\sigma}$ in $\operatorname{Reg}(\mu)$ and $A_{\delta}$ a $G_{\delta}$ in $\operatorname{Reg}(\mu)$, so that $A_{\sigma} \subset A \subset A_{\delta}$ and so that $\mu\left(A_{\delta} \backslash A_{\sigma}\right)=$ $\mu_{\text {reg }}\left(A_{\delta} \backslash A_{\sigma}\right)=0$. Let $\left\{A^{\sigma}, A^{\delta}\right\} \subset \mathscr{B}_{0} \cap \operatorname{Reg}(\mu)$ with $A_{\sigma} \subset A^{\sigma} \subset A^{\delta} \subset A_{\delta}$. Then, $\mu(A)=\mu\left(A^{\sigma}\right)=\mu_{\mathrm{reg}}\left(A^{\sigma}\right)=\mu_{\mathrm{reg}}(A)$.

In the remainder of the paper we will be dealing fairly exclusively with extensions $\mu$ of Dirac measures $\delta_{x}$ for $x \in X$ from $\mathscr{B}_{0}$ to $\mathscr{G}_{\text {. All such extensions }}$ must be $\{0,1\}$-valued on $\operatorname{Reg}(\mu)$. If $A \in \operatorname{Reg}(\mu)$ then $\mu(A)=0$ if and only if $x \notin A$.

PROPOSITION 6. Let $\Sigma_{1} \subset \Sigma_{2}$ be $\sigma$-algebras of subsets of a set $\Omega$. Let $\delta \in C A_{1}^{+}\left(\Sigma_{1}\right)$ be $\{0,1\}$-valued. Let $\eta$ be the $\sigma$-ideal in $\Sigma_{2}$ of sets of outer measure 0 under $\delta$.

i) If the quotient algebra $\Sigma_{2} / \eta$ is finite then $B A_{1}^{+}\left(\Sigma_{1}, \delta, \Sigma_{2}\right)$ is a finite dimensional subset of $C A_{1}^{+}\left(\Sigma_{2}\right)$.

ii) If $\Sigma_{2} / \eta$ is infinite there is a family $\left\{\mu_{t}^{l}\right\} \subset B A_{1}^{+}\left(\Sigma_{1}, \delta, \Sigma_{2}\right)$ of mutually singular, non-atomic, purely finitely additive measures whose cardinality is $2^{c}$ where $c$ is the continuum.

Proof. There is an affine bijection from $B A_{1}^{+}\left(\Sigma_{1}, \delta, \Sigma_{2}\right)$ to $B A_{1}^{+}\left(\Sigma_{2} / \eta\right)$. If $\mu \in B A_{1}^{+}\left(\Sigma_{1}, \delta, \Sigma_{2}\right)$ then $\mu(A)=0$ for all $A \in \eta$ hence $\mu$ induces on $\Sigma_{2} / \eta$ an element, also denoted by $\mu$, in the usual fashion. This gives the affine bijection.

ii) If $\Sigma_{2} / \eta$ is infinite it is an infinite $F$-algebra as in [3]. By Corollary 3.2.3 of [3] there is a family $\left\{\mu_{t}\right\}$, of cardinality $2^{c}$, of mutually singular non-atomic probability measures on $\Sigma_{2} / \eta$ all with the same negligible sets. Pulling back under the affine bijection from $B A_{1}^{+}\left(\Sigma_{1}, \delta, \Sigma_{2}\right)$ to $B A_{1}^{+}\left(\Sigma_{2} / \eta\right)$ one obtains the same sort of family in $B A^{+}\left(\Sigma_{1}, \delta, \Sigma_{2}\right)$. If $\mu_{s} \in\left\{\mu_{t}\right\}$ is countably additive there can be no other countably additive $\mu_{r} \in\left\{\mu_{t}\right\}$ for $\mu_{r} \perp \mu_{s}$ and both have the same nullsets. Delete $\mu_{s}$ if necessary so that no element of $\left\{\mu_{t}\right\}$ is countably additive. Each $\mu_{t}$ has a non-trivial purely finitely additive part which is a multiple of a purely finitely additive $\mu_{t}^{\prime}$ which is easily verified to belong to $B A_{1}^{+}\left(\Sigma_{1}, \delta, \Sigma_{2}\right)$. Furthermore, $\mu_{t}^{\prime}$ must be non-atomic for each $t$. This establishes ii). 
i) Suppose that $\Sigma_{2} / \eta$ is finite and has $n$ atoms $\left\{a_{1}, \ldots, a_{n}\right\}$. Corresponding to each $a_{i}$ is an $A_{i} \in \Sigma_{2}$ which is such that if $A \in \Sigma_{2}$ then $A_{i} \backslash A \in \eta$ or $A \cap A_{i} \in \eta$. The $\{0,1\}$-valued measure $\delta_{i}$ on $\Sigma_{2} / \eta$ or in $B A_{1}^{+}\left(\Sigma_{1}, \delta, \Sigma_{2}\right)$ corresponding to $a_{i}$ is an extreme point of $B A_{1}^{+}\left(\Sigma_{2} / \eta\right)$ and $B A_{1}^{+}\left(\Sigma_{2} / \eta\right)=\operatorname{conv}\left(\delta_{1}, \ldots, \delta_{n}\right)$. To show that $B A_{1}^{+}\left(\Sigma_{1}, \delta, \Sigma_{2}\right) \subset C A^{+}\left(\Sigma_{2}\right)$ it suffices to show that each $\delta_{i}$, considered as an element of $B A_{1}^{+}\left(\Sigma_{1}, \delta, \Sigma_{2}\right)$, is in $C A^{+}\left(\Sigma_{2}\right)$. To this end let $\left\{E_{n}\right\}$ be an increasing sequence in $\Sigma_{2}$ with $\delta_{i}\left(E_{n}\right)=0$ for all $n$. We have $E_{n} \cap A_{i} \in \eta$ for all $n$ hence, by the $\sigma$-completeness of $\eta$, we have $\left(\cup_{n} E_{n}\right) \cap A_{i} \in \eta$. Thus, $\delta_{i}\left(\cup_{n} E_{n}\right)$ $=0$. This establishes countable additivity of $\boldsymbol{\delta}_{i}$ hence establishes i).

Remarks. Recall from [2] that a measure $\mu$ is strongly finitely additive if and only if there is a partition $\left\{A_{n}: n \in N\right\}$ with $\mu\left(A_{n}\right)=0$ for all $n$. Any purely finitely additive probability measure is the sum of countably many strongly finitely additive measures, [2]. In ii) purely finitely additive measures may be replaced by strongly finitely additive measures.

Actually ii) asserts only that such a family of probabilities exists in $B A\left(\Sigma_{2} / \eta\right)$. This is true if $\eta$ is replaced by the ideal generated by the null sets of a non $\{0,1\}$-valued measure or $\Sigma_{2} / \eta$ by an arbitrary $F$-algebra.

COROLlary 6.1. If $\Sigma_{2} / \eta$ is infinite there exist c purely finitely additive $\{0,1\}$-valued elements of $B A_{1}^{+}\left(\Sigma_{1}, \delta, \Sigma_{2}\right)$.

Proof. There is a strongly finitely additive non-atomic $\mu \in B A_{1}^{+}\left(\Sigma_{1}, \delta, \Sigma_{2}\right)$. Let $\left\{A_{n}\right\} \subset \Sigma_{2}$ be an increasing sequence with $\mu\left(A_{n}\right)=0$ for all $n$ and with $\cup_{n} A_{n}=\Omega$. Let $\mathscr{Q}$ denote the algebra $\Sigma_{2} / \eta$ and let $X_{\mathfrak{Q}}$ be its Stone space. $B A_{1}^{+}\left(\Sigma_{1}, \delta, \Sigma_{2}\right)$ is affinely homeomorphic to the Bauer simplex of Radon probability measures on $X_{\mathbb{Q}}$. Let $\tilde{\mu}$ be the Radon measure on $X_{\mathbb{Q}}$ corresponding to $\mu$ so that if $A \in \Sigma_{2} / \eta$ or if $A \in \Sigma_{2}$ then $\mu(A)=\tilde{\mu}([A])$ where $[A]$ is the clopen set in $X_{\Theta}$ corresponding to $A$. We have $\mu(A)=\int \chi_{[A]}(x) \tilde{\mu}(d x)=\int \chi_{x}(A) \tilde{\mu}(d x)$ (where $x \in X_{\mathscr{Q}}$ are considered as ultrafiters on $\mathscr{Q}$ ). If there were a set $Z$ with outer measure $\tilde{\mu}^{*}(Z)>0$ of $\delta$-ultrafilters $x \in X_{\mathscr{Q}}$ (so that each $\chi_{x}$ is countably additive on $Q$ ), it would follow that $0=\lim _{n \rightarrow \infty} \mu\left(A_{n}\right)=\lim _{n \rightarrow \infty} \int \chi_{\left[A_{n}\right]}(x) \tilde{\mu}(d x) \geqslant$ $\tilde{\mu}^{*}(Z)>0$. Since this is impossible $\tilde{\mu}$-almost all $x \in X_{Q}$ have $\chi_{x}$ purely finitely additive. Since $\tilde{\mu}$ is non-atomic there is a compact perfect set $Y \subset \operatorname{supp}(\tilde{\mu}) \subset X_{\mathscr{Q}}$ so that if $x \in Y$ then $\chi_{x}$ is purely finitely additive. $Y$ contains at least $c$ elements.

COROLlaRY 6.2. If $\mathfrak{Q}$ is a Boolean algebra then $\mu \in B A_{1}^{+}(\mathbb{Q})$ is purely finitely additive with corresponding measure $\tilde{\mu}$ on the Stone space $X_{\mathscr{Q}}$ only if $\mu$-almost all $x \in X_{\mathbb{Q}}$ are not $\delta$-ultrafilters. 
We may apply the preceding results to the case where $\mathscr{B}_{0}=\Sigma_{1}$ and $\mathscr{B}=\Sigma_{2}$. A $\{0,1\}$-valued measure $\delta$ on $\mathscr{B}_{0}$ is a Dirac measure $\delta_{x}$. $\eta$ will be denoted by $\eta_{x} \cdot \eta_{x}$ consists of those Borel sets in $X$ contained in a $\sigma$-compact subset of $X^{\prime}=X \backslash\{x\}$. We are only interested in the case where $\mathscr{B} / \eta_{x}=\mathscr{B}_{x}$ has cardinality larger than 2 so that $\{x\}$ is not a $G_{\delta}$.

Proposition 7. Let $x$ be a non- $G_{\delta}$-point in $X$.

i) If $\mathscr{B}_{x}$ is finite the elements of $B A_{1}^{+}\left(\mathscr{B}_{0}, \delta_{x}, \mathscr{G}_{3}\right)$ form a finite dimensional simplex in $C A_{1}^{+}(\mathscr{B})$. In this case there is a $\mu \in B A_{1}^{+}\left(\mathscr{B}_{0}, \delta_{x}, \mathscr{B}_{)}\right)$with $\operatorname{Reg}(\mu)=$ $\hat{\mathscr{B}}_{0}^{\delta_{x}}=\hat{\mathscr{B}}_{0}^{\mu}$.

ii) If $\mathscr{B}_{x}$ is infinite there is a family of cardinality $2^{c}$ of singular non-atomic purely finitely additive elements of $B A_{1}^{+}\left(\mathscr{G}_{0}, \delta_{x}, \mathscr{B}\right)$ and a family of cardinality $c$ of $\{0,1\}$-valued purely finitely additive elements.

Proof. We need only find in case i) a $\mu \in B A_{1}^{+}\left(\mathscr{G}_{0}, \delta_{x}, \mathscr{B}\right)$ with $\operatorname{Reg}(\mu)=\hat{\mathscr{B}}_{0}^{\mu}$. Let $\left\{\delta_{x}, \delta_{1}, \ldots, \delta_{n}\right\}$ denote the extreme points of $B A_{1}^{+}\left(\mathscr{B}_{0}, \delta_{x}, \mathscr{B}\right)$ where $\delta_{x}$ is the usual Dirac measure on $\mathscr{B}$. We assert that $\mu=\frac{1}{n}\left(\delta_{1}+\cdots+\delta_{n}\right)$ has $\operatorname{Reg}(\mu)=\hat{\mathscr{B}}_{0}^{\mu}$. Suppose not. Note that $\hat{\mathscr{B}}_{0}^{\mu}=\hat{\mathscr{B}}_{0}^{\delta_{x}}$ is the largest subalgebra of $\mathscr{B}$ to which $\delta_{x}$ has a unique extension. Note also that $\delta_{x}$ agrees with $\mu$ on $\operatorname{Reg}(\mu)$ by Proposition 5 . There is an extreme extension $\delta$ of $\delta_{x}$ from $\hat{\mathscr{B}}_{0}^{\mu}$ to $\operatorname{Reg}(\mu)$ other than $\delta_{x}$ hence other than $\mu$. This extreme extension $\delta$ is the restriction of one of $\left\{\delta_{1}, \ldots, \delta_{n}\right\}$ to $\operatorname{Reg}(\mu)$, say $\delta_{1}$. Since all extreme extensions of $\delta_{x}$ to $\operatorname{Reg}(\mu)$ are $\{0,1\}$-valued there is an $A \in \operatorname{Reg}(\mu)$ with $0=\delta_{x}(A)=\mu(A)$ and $\delta_{1}(A)=1$. But $\mu(A)=$ $\frac{1}{n}\left(\delta_{1}(A)+\cdots+\delta_{n}(A)\right) \geqslant \frac{1}{n}$ which is impossible. Thus, $\operatorname{Reg}(\mu)=\hat{\mathscr{B}}_{0}^{\mu}$.

Corollary 7.1. If $\mathscr{B}_{x}$ is infinite and $\mu \in B A_{1}^{+}\left(\mathscr{G}_{0}, \delta_{x}\right.$, $\left.\mathscr{B}\right)$ has $\operatorname{Reg}(\mu) \neq \hat{\mathscr{B}}_{0}^{\mu}$ there is a $\nu \in B A_{1}^{+}\left(\mathscr{B}_{0}, \delta_{x}, \Re_{B}\right)$ with $\operatorname{Reg}(\nu)$ a proper subset of $\operatorname{Reg}(\mu)$.

REMARK. We know of no case in which $x$ is a non- $G_{\delta}$-point for which i) holds in Proposition 7. For the case $X=\left[0, \omega_{1}\right]$ and $x=\omega_{1}$ one may set $A_{0}$ equal to the relatively closed set in $\left[0, \omega_{1}\right)$ consisting of limit ordinals, and set $A_{n}=\{\alpha+1$ : $\left.\alpha \in A_{n-1}\right\}$ for $n \in \omega$. Then $\left[0, \omega_{1}\right)=\cup_{n} A_{n}$. Each $A_{n}$ is in $\mathscr{B}_{\backslash} \backslash \eta_{x}$ hence $\mathscr{B}_{x}$ is infinite. A similar argument shows that if $D$ is an infinite discrete set with uncountable cardinality then $X=D \cup\{\infty\}$ has $\mathscr{B}_{x}$ infinite then $x=\infty$.

COROLlaRY 7.2. If $\mathscr{B}_{x}$ is finite there is a closed set $E \subset X^{\prime}$ whose complement is o-compact and is such that $E$ has a partition $\left\{E_{1}, \ldots, E_{n}\right\}$ with each $E_{i}$ closed. Within each $E_{i}$ the set $\bar{F}_{i}$ of non-o-compact closed sets forms a $\delta$-ultrafilter of closed sets. If $E_{i} \cup\{x\}=X_{i}$ is considered as the one point compactification of $E_{i}$ then $\delta_{x}$ 
has a one dimensional simplex of extensions to the Borel sets of $X_{i}$. The extreme extension $\delta_{i}$ is defined by $\delta_{i}(A)=1$ if and only if $A$ contains an element of $\mathscr{F}_{i}$ for $i=1, \ldots, n$.

PRoof. Let $\left\{\delta_{0}, \delta_{1}, \ldots, \delta_{n}\right\}$ be the extreme elements of $B A_{1}^{+}\left(\mathscr{B}_{0}, \delta_{x}, \mathscr{B}\right)$ with $\delta_{0}$ the regular extension. For each $i=1, \ldots, n$ there is a $\delta$-ultrafilter $\mathscr{F}_{i}$ of closed subsets of $X^{\prime}$ so that $\delta_{i}(A)=1$ if and only if $A$ meets each element of $\mathscr{F}_{i}$. One may find $\left\{F_{1}, \ldots, F_{n}\right\}$ so that $F_{i} \in \mathscr{F}_{i}$ for $i=1, \ldots, n$ and so that $F_{i} \cap F_{j} \in \eta_{x}$ for all $i \neq j$. One may find an open o-compact $\theta \subset X^{\prime}$ with $F_{i} \cap F_{j} \subset \theta$ for all $i, j$. Let $E_{i}=F_{i} \backslash \theta$ for all $i$ and let $E=\cup_{i=1}^{n} E_{i}=X^{\prime} \backslash \theta$. Any extension $\delta$ of $\delta_{x}$ to the Borel sets of $X_{i}$ with $\delta(x)=0$ may be extended to an element of $B A_{1}^{+}\left(\mathscr{B}_{0}, \delta_{x}, \mathscr{B}\right)$ with $\delta\left(E_{i}\right)=1$. We must have $\delta=\delta_{i}$ which establishes the corollary.

COROLLARY 7.3. If $\Re_{x}$ is finite every closed set in $X^{\prime}$ contains a dense $\sigma$-compact subset.

Proof. We may, by Corollary 7.2 , assume that $B A_{1}^{+}\left(\mathscr{B}_{0}, \delta_{x}, \mathscr{B}\right)=\left\{\delta_{x}, \delta\right\}$ so that $\tilde{F}=\left\{F\right.$ closed in $\left.X^{\prime}: \delta(F)=1\right\}$ is the set of non- $\sigma$-compact closed sets in $X^{\prime}$.

Assume that $X^{\prime} \neq \bar{E}$ for any $E \in \eta_{x}$. If this is the case then $E \in \eta_{x}$ implies that $\bar{E} \in \eta_{x}$. To see this note that if $\bar{E} \notin \eta_{x}$ then $\bar{E} \in \mathscr{F}$ and $\bar{E}^{\mathrm{c}} \in \eta_{x}$. Since $X$ is the closure of $E \cup \bar{E}^{c} \in \eta_{x}$ one has a contradiction.

Let $\left\{\theta_{\alpha}\right\} \subset \eta_{x}$ be a sequence indexed by ordinals $\alpha$ defined by transfinite induction so that $\bar{\theta}_{\alpha}$ is a proper subset of $\theta_{\alpha+1}$ and so that $\theta_{\alpha}=\cup_{\beta<\alpha} \theta_{\beta}$ if $\alpha$ is a limit ordinal. The last element $\theta_{\lambda}$ of this sequence occurs for a limit ordinal $\lambda$ so that $\bar{\theta}_{\lambda} \in \mathscr{F}$ hence so that $\theta_{\lambda} \notin \eta_{x}$. Since $\eta_{x}$ is $\sigma$-complete $\lambda$ is of uncountable cofinality. Let $\psi_{\alpha}=\theta_{\alpha+1} \backslash \bar{\theta}_{\alpha}$ for $\alpha<\lambda$ and let $\psi_{\lambda}=X^{\prime} \backslash \vec{\theta}_{\lambda}$. We have $X^{\prime}=$ $\left[\cup\left\{\psi_{\alpha}: \alpha \leqslant \lambda\right\}\right] \cup\left[\cup\left\{\partial \theta_{\alpha}: \alpha<\lambda\right\}\right]$. The open set $\cup\left\{\psi_{\alpha}: \alpha \leqslant \lambda\right\}$ is dense in $X^{\prime}$ hence is not in $\eta_{x}$. The closed set $\cup\left\{\partial \theta_{\alpha}: \alpha<\lambda\right\}$ is $\sigma$-compact hence is in an open $\theta_{\infty} \in \eta_{x}$. Let $D=\left\{\alpha \leqslant \lambda: \psi_{\alpha} \backslash \theta_{\infty} \neq \varnothing\right\}$. The open sets $\left\{\psi_{\alpha}: \alpha \in D\right\}$ together with $\theta_{\infty}$ cover $X^{\prime}$. Thus, $\operatorname{card}(D) \geqslant \boldsymbol{\kappa}_{1}$. If $K$ is a compact set in $X^{\prime}$ it is covered by $\theta_{\infty}$ together with finitely many $\psi_{\alpha}$ with $\alpha \in D$ hence a $\sigma$-compact set is covered by $\theta_{\infty}$ together with countably many $\psi_{\alpha}$ with $\alpha \in D$. Let $\left\{D_{n}: n \in N\right\}$ be a countable partition of $D$ into uncountable sets. For each $n$ let $U_{n}=\bigcup\left\{\psi_{\alpha}: \alpha \in D_{n}\right\}$. The family $\left\{U_{n}: n \in N\right\}$ is a disjoint family of open sets with $\bigcup\left\{U_{n}: n \in N\right\}=\bigcup\left\{\psi_{\alpha}\right.$ : $\alpha \in D$. Since a $\sigma$-compact $F$ meets only countably many $\psi_{\alpha}$, no $U_{n}$ is in $\eta_{x}$. Thus, $\mathscr{B}_{x}$ is infinite which is impossible. Thus, $X^{\prime}=\bar{E}$ for some $E \in \eta_{x}$. This demonstration also establishes, if $F \in \mathscr{F}$ replaces $X^{\prime}$, that $F=\bar{E}$ for some $E \in \eta_{x}$, which establishes the corollary. 
In the unlikely event that $\mathscr{B}_{x}$ be finite for some non- $G_{\delta}$-point $x$, Proposition 7 gives a countably additive $\mu \in B A_{1}^{+}\left(\mathscr{B}_{0}, \delta_{x}, \mathscr{B}\right)$ with $\operatorname{Reg}(\mu)=\hat{\mathscr{B}}_{0}^{\mu}$. We conclude by giving an example where $\operatorname{Reg}(\mu)$ is always larger than $\hat{\mathscr{P}}_{0}^{\mu}$.

EXAMPLE 8. Let $X$ be the one point compactification $D \cup\{x\}$ of an uncountable discrete space. $\mathscr{B}_{0}$ consists of countable sets in $D$ and their complements in $X$, $\mathscr{B}=2^{X}$ and $\eta_{x}$ consists of countable sets in $D$ hence is a maximal ideal in $\mathscr{B}_{0}$ and $\mathscr{B}_{0}$ is $\mu$-complete for any $\mu \in B A_{1}^{+}\left(\mathscr{G}_{0}, \delta_{x}, \mathscr{B}\right)$. The $\mu \in B A_{1}^{+}\left(\mathscr{G}_{0}, \delta_{x}, \mathscr{B}\right)$ with $\mu(\{x\})=0$ are identified with elements of $B A_{1}^{+}\left(2^{D} / \eta_{x}\right)$ or with elements of $B A_{1}^{+}\left(2^{D}\right)$ which annihilate $\eta_{x}$ hence are those $\mu \in B A_{1}^{+}\left(2^{X}\right)$ with $\mu(A)=0$ if $A$ is countable in $X$. If $\mu \in B A_{1}^{+}\left(\mathscr{B}_{0}, \delta_{x}, \mathscr{B}\right)$ then $\mu$ agrees with $\delta_{x}$ on $\operatorname{Reg}(\mu)$. If $A \subset D$ has $\mu(A)=0$ then $A \in \operatorname{Reg}(\mu)$ since $A$ is open whereas $A \cup\{x\} \notin$ $\operatorname{Reg}(\mu)$. Thus, $\operatorname{Reg}(\mu)$ consists of $A \subset D$ with $\mu(A)=0$ and the complements in $X$ of these $A$. Let $\eta_{\mu}$ denote the ideal in $2^{D}$ of $\mu$-negligible sets. $\eta_{\mu}$ is a maximal ideal in $\operatorname{Reg}(\mu)$ and $2^{D} / \eta_{\mu}$ satisfies the countable chain condition. On the other hand $2^{D} / \eta_{x}$ does not satisfy the countable chain condition since $D$ has an uncountable partition into uncountable sets. Thus, $\eta_{x} \neq \eta_{\mu}$ and $\hat{\mathscr{G}}_{0}^{\mu} \neq \operatorname{Reg}(\mu)$.

Note that if the cardinality of $D$ is not real-valued measurable, [1], [2], then all elements $\mu$ of $B A_{1}^{+}\left(\Re_{0}, \delta_{x}, B_{B}\right)$ with $\mu(\{x\})=0$ must be purely finitely additive. If the cardinality of $D$ is real-valued measurable any countably additive diffuse measure $m$ on $2^{D}$ gives an element of $C A_{1}^{+}\left(\mathscr{B}_{0}, \delta_{x}, \mathscr{S}_{3}\right)$ singular to $\delta_{x}$ and $\operatorname{Reg}(\mu)$ is guaranteed to be strictly between $\mathscr{B}_{0}$ and $\mathscr{B}$. If $\mu \in B A^{+}\left(\mathscr{B}_{0}, \delta_{x}, \mathscr{B}\right)$ is purely finitely additive it is a countable convex combination $\sum\left\{\lambda_{n} \mu_{n}: n \in \mathbf{N}\right\}$ of strongly finitely additive $\left\{\mu_{n}\right\} \subset B A_{1}^{+}(\mathscr{B})$. Each $\mu_{n}$ must be in $B A_{1}^{+}\left(\mathscr{B}_{0}, \delta_{x}, \mathscr{B}\right)$. From the definition of strong finite additivity there exist $\left\{A_{m}^{n}: m \in \mathbf{N}\right\} \subset \eta_{\mu_{n}}$ which partition $D$. We have $\left\{A_{m}^{n}: m \in \mathbf{N}\right\} \subset \operatorname{Reg}\left(\mu_{n}\right)$. Since $D \notin \operatorname{Reg}\left(\mu_{n}\right)$ it is impossible for $\operatorname{Reg}\left(\mu_{n}\right)$ to be $\sigma$-algebra even though $\mathscr{B}_{0} \subset \operatorname{Reg}\left(\mu_{n}\right)$.

Remark. Karel Prikry and Richard Gardner pointed out Example 8.

\section{References}

[1] T. E. Armstrong and K. Prikry, 'Residual measures', Illinois J. Math. 22 (1978), 64-78.

[2] T. E. Armstrong and K. Prikry, ' $\kappa$-finiteness and $\kappa$-additivity of measures on sets and left-invariant measures on discrete groups', Proc. Amer. Math. Soc. 80 (1980), 105-112.

[3] T. E. Armstrong and K. Prikry, 'Liapounoff's theorem for non-atomic bounded, finitely additive, finite dimensional, vector valued measures', Trans. Amer. Math. Soc. 266 (1981). 499-514.

[4] A. G. A. G. Babiker and J. D. Knowles, 'An example concerning completion regular measures. images of measurable sets and measurable selections', Mathematika 25 (1978), 120-124.

[5] S. K. Berberian, Measure and integration, (Chelsea, New York, 1970). 
[6] P. Billingsley, Convergence of probability measures, (Wiley, New York, 1968).

[7] F. Dashiell, A. Hager and M. Henriksen, 'Order-Cauchy completions of rings and vector lattices of continuous functions', Canad. J. Math. 32 (1980), 657-685.

[8] R. J. Gardner, 'The regularity of Borel measures and Borel measure compactness', Proc. London Math. Soc. 30 (1975), 95-113.

[9] L. Gillman and M. Jerison, Rings of continuous functions, (Van Nostrand, Princeton, N. J., 1960).

[10] W. Graves and R. F. Wheeler, 'On the Grothendieck and Nikodym properties for algebras of Baire, Borel and universally measurable sets', preprint.

[11] P. Halmos, Measure theory, (Van Nostrand, Princeton, N. J., 1950).

[12] P. Halmos, Lectures on Boolean algebras, (Van Nostrand, Princeton, N. J., 1963).

[13] E. Hewitt and K. Yosida, 'Finitely additive measures', Trans. Amer. Math. Soc. 72 (1952), 46-66.

[14] K. Jacobs, Measure and integral, (Academic Press, New York, 1978).

[15] J. L. Kelley, General topologv, (Van Nostrand, Princeton, N. J., 1955).

[16] J. Kupka, 'Uniform boundedness principles for regular Borel vector measures', J. Austral. Math. Soc. 29 (1980), 206-218.

[17] R. Levy, 'Almost P-spaces', Canad. J. Math. 29 (1977), 284-288.

[18] A. F. Martin, 'A note on monogenic Baire measures', Amer. Math. Monthly 84 (1977), 554-555.

[19] S. Okada and Y. Okasaki, 'On measure-compactness and Borel measure-compactness', Osaka J. Math. 15 (1978), 183-191.

[20] D. Plachky, 'Extremal and monogenic additive set functions', Proc. Amer. Math. Soc. 54 (1976), 193-196.

[21] W. Schachermayer, 'Eberlein-compacts et espaces de Radon', Bol. Ann. Sci. Univ. Clermont 61 (1976), 129-145.

[22] W. Schachermayer, 'On compact spaces which are not $c$-spaces', Bol. Soc. Mat. Mexicana 22 (1977), 60-63.

[23] Z. Semadeni, Banach spaces of continuous functions, (Polish Scientific Press, Warsaw, 1971).

[24] R. Sikorski, Boolean algebras, (Springer, New York, 1969).

[25] F. Topsoe, 'Approximating pavings and construction of measures', Collog. Math. 421979. 377-385.

[26] T. Traynor, 'The Lebesgue decomposition for group valued set functions', Trans. Amer. Math. Soc. 220 (1976), 307-319.

[27] A. E. Veksler, 'Functional characteristics of $P^{\prime}$-spaces', Comment. Math. Univ. Carolinae 18 (1977), 363-366.

[28] R. C. Walker, The Stone- $\breve{C} e c h$ compactification, (Springer, New York, 1974).

\section{Department of Mathematical Sciences}

Northern Illinois University

De Kalb, Illinois 60115

U.S.A. 